\title{
A Practical Method for Purification of Fat Grafts Using Gelatin Sponge
}

\section{Jelatin Sünger Kullanarak Yağ Greftlerinin Saflaştırılması için Pratik Bir Yöntem}

\section{(D) Perçin Karakol1, (D) Mehmet Bozkurt1, (D) Emin Kapı²}

\author{
${ }^{1}$ Health Science University Turkey, Bağcılar Training and Research Hospital, Clinic of Plastic, Reconstructive and Aesthetic Surgery, İstanbul, Turkey \\ ${ }^{2}$ Adana Training and Research Hospital, Clinic of Plastic, Reconstructive and Aesthetic Surgery, Adana, Turkey
}

\section{Dear Editor,}

Fat grafting has dramatically become popular during recent years not only in plastic surgery, but also in other medical fields. Processing of fat grafts can be done in different ways, either with filtering, cotton-gauze rolling, or centrifugation methods (1). A major focus was the use of centrifugation or other methods to separate the aqueous and oil components before injection because this stage is the most important part for fat graft survival (2). There is no consensus on the various techniques, neither a gold standard technique for processing stage. In this letter, we aimed to use an alternative method for purification and filtering of fat grafts using gelatin sponge for low quantities of fat grafts.

Our standard tumescent solution is injected into the harvest site. Fat is aspirated by hand using $60 \mathrm{~mL}$ syringe and harvesting cannula. In this method, fat lobules are removed through fibrous septas by degrading. For this reason, fat grafts obtained are free of fibrous septa and have a homogenic formation. A gelatin sponge with dimension of $80 \times 50 \times 10 \mathrm{~mm}$ is divided transversely into two parts by using no: 10 blades for enlarging the surface area used (Figure 1a and $b$ ). The rough surfaces of the gelatin sponge are used as it is more absorbent. The hand-suctioned specimens are divided into lesser parts and poured onto the surface of the sponge. The specimens are rolled with forceps for about 3 minutes until all blood, aqueous and oil components are absorbed and separated (Figure $1 \mathrm{c}$ and d). The purified fat grafts area are filled to $2 \mathrm{~mL}$ syringes using curettes. The purified and unprocessed fat graft is seen in Figure 2.

The gelatin sponge for purifying fat grafts is a cheap, quick method and it is quite easy to apply. Gelatin sponge (Spongostan ${ }^{\circledR}$, Ferrosan A/S, Denmark) is an absorbable hemostatic agent derived from pig skin collagen and is widely used in humans for over 60 years (3). It is a material that can be absorbed between 4 and 6 weeks, and it is sponge-like and contains hemostatic gelatin. It has porous formation. It promotes platelet aggregation and increases the matrix formation of fibrin (4). It is cheap $(0.30 €$ per one) and commercially available, it can be found easily almost in every hospital. The surgeon himself can easily perform the technique without any help. Although fat grafting which is performed by closed system has been regarded as ideal, there has not been any kind of evidence that indicates infection is increased through desiccation air oxidation or decreases fat graft viability (1). As far as the study of the Kuran and Tumerdem (5), although the application period is 15-20 minutes, there have been available data showing that air exposure does not increase bacterial growth. In our study, as gelatin sponge has a fast absorbent character,

Address for Correspondence: Perçin Karakol, Health Science University Turkey, Bağcılar Training and Research Hospital, Clinic of Plastic,

Reconstructive and Aesthetic Surgery, İstanbul, Turkey

E-mail: ppercin@gmail.com ORCID ID: orcid.org/0000-0003-0068-2139 Received: 06.02.2020 Accepted: 21.04 .2020

Cite this article as: Karakol P, Bozkurt M, Kapı E. A Practical Method for Purification of Fat Grafts Using Gelatin Sponge. Bagcilar Med Bull 2020;5(2):64-66

(C) Copyright 2020 by the Health Sciences University Turkey, Bagcilar Training and Research Hospital

Bagcilar Medical Bulletin published by Galenos Publishing House. 
execution time is shorter and thus we believe that potential risks are minimized. The common agreed methods for fat processing are the cotton gauze rolling and centrifugation, and also filtration which is less commonly used. According to literature, there are conflicting reports on that one of them has superiority to another $(1,6)$. The filtration method has an advantage to process large volumes efficiently and quickly at the same time. It may be safer for infection and
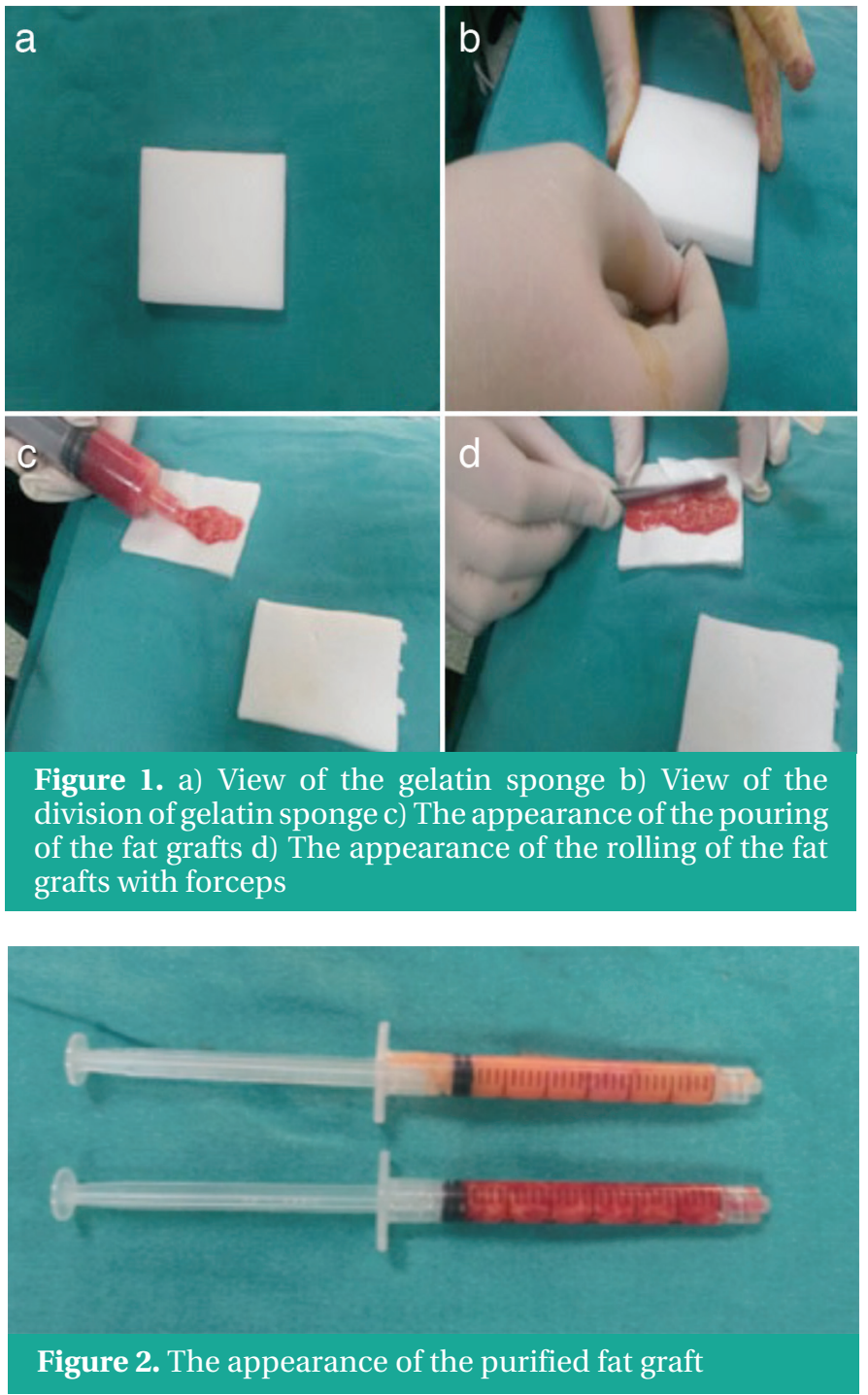

it increases graft viability but there is not enough evidence for these situations. It is not widely available, not suitable for little volumes; and it is also expensive (1). Kuran and Tumerdem (5) also described a filtration method which was inspired by yogurt concentration methods in Anatolia, that is believed to prevent the adverse affects of centrifugation (5).

The centrifugation and cotton gauze rolling methods are useful for little amounts of fat. The cotton gauze derived technique of Telfa (Covidien, Mansfield, Mass.) is commercially available in local market. It is disposable. It is proven to be the best option for removing oil fraction (1). The centrifugation method (Coleman's technique) is as efficient to purify and to drain fat by gravity as the cotton gauze method. It can be found easily but it requires a centrifugation machine and cannot be performed by the surgeons themselves during the operation.

In the comparison of filtering, centrifugation, cotton gauze rolling and gelatin sponge methods, the processing time, residual oil ratio contained in the graft after processing, removing of aqueous oil degree, the fat grafts volume retention percentile which was observed in 6 weeks after the application were compared (Table 1). According to this, in the cotton gauze sponge and gelatin sponge techniques, it was seen that both period of time was shortened and the most favorable results were obtained in terms of efficacy.

Compared to all groups, in the gelatin sponge group, adipocytes were evaluated by examining the presence of needle-shaped fissure formations, which contain nuclei in terms of viability and the radial distribution formed by dissolving the triglyceride crystals. In terms of this parameter, adipocyte viability was found to be high in fat graft samples. It is noteworthy that fibrotic areas are in narrower areas, and capillary blood vessels are much denser among adipocytes. Minimal necrosis was observed when staining with hematoxylin eosin (Figure 3).

As the processes of cotton gauze and filtration, a drain work is performed by making the fat grafts held or mixed.

Table 1. Comparison of filtering, centrifugation, cotton gauze rolling in gelatin sponge methods

\begin{tabular}{lllll} 
& Methods & & & \\
\cline { 2 - 5 } Parameters & Filtering & Centrifugation & Cotton gauze rolling & Gelatin sponge \\
\hline Processing time (min) & 5 & 3 & 5 & 0 \\
Residual oil (\%) & 1 & 4 & +++ & +++ \\
Removing of aqueous oil & ++ & ++ & 70 & 75 \\
Fat graft volume retention (\%) & 58 & 47 & & + \\
\hline
\end{tabular}

min: Minimum 


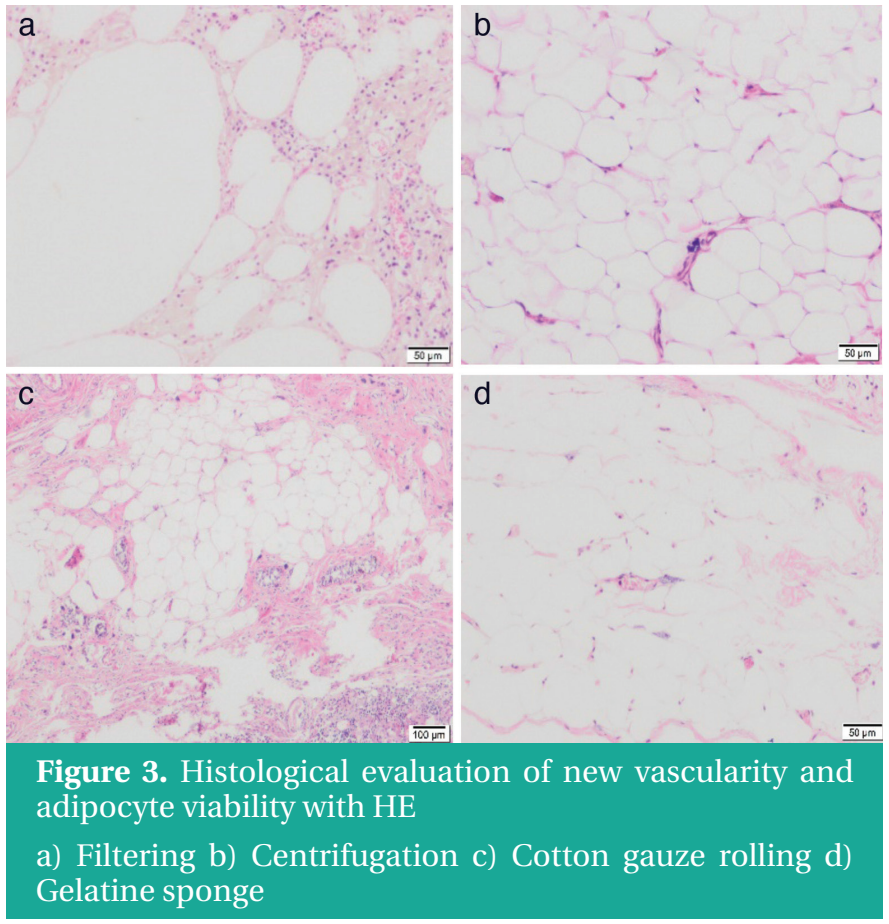

In spite of a long holding duration for these works, it can be faced by the situations that the liquid side of the fat is not absorbed efficiently. As the content of gelatin sponge is of the characteristic for rather absorbing and spongeporous, it absorbs the liquid quickly and it makes fat graft concentrated. In addition to this, the possibility that gauze particles may remain between the fat tissues in cotton gauze method, it brings about the foreign-body reaction risk. Since gelatin sponge is of the kind that melts, we are of the opinion that the tendency of forming foreign-body reaction in the long term period is lower. At the same time, besides mechanical effect over the fat graft application, because of the contribution to fibrin formation, we think that fat grafts increase the penetration into tissue and decreases the melting rate (5).

The most important limitation of this method is that it allows little amounts of fat purification. A single gelatin sponge can purify about $10 \mathrm{~mL}$ of fat and about 3-4 $\mathrm{mL}$ purified fat graft is obtained. We use purified fat grafts into facial areas especially nasolabial sulcus and malar area and the obtained amounts are usually sufficient. If greater amounts are required, it may not be favorable, because of timely and personal constraints; other purification techniques like filtration may be used. Further studies must be performed for the effectiveness and showing the advantages and disadvantages and short and long term results may be helpful for comparison. For the parameters which are the matters in question, performing experimental work is more convenient. For this issue, a more detailed study has been planned.

Although, the cotton gauze rolling and centrifugation have become established methods for removing unwanted oil and aqueous fluid from the lipo-aspirate to purify the fat before grafting, the presented gelatin sponge method may be kept in mind as an alternative due to its easiness, cheapness especially for small amounts of fat grafts. The manuscript does not contain clinical studies or patient data.

Keywords: Fat graft, sponge, purification

Anahtar kelimeler: Saflaştırma, sünger, yağ grefti

\section{Acknowledgement}

Thanks to Emre Güvercin MD for his contributions.

\section{Ethics}

Peer-review: Externally peer-reviewed.

\section{Authorship Contributions}

Surgical and Medical Practices: M.B., Concept: P.K., Design: P.K., Data Collection or Processing: P.K., M.B., Analysis or Interpretation: E.K., Writing: P.K., M.B.

Conflict of Interest: No conflict of interest was declared by the authors.

Financial Disclosure: The authors declared that this study received no financial support.

\section{References}

1. Fisher C, Grahovac TL, Schafer ME. Comparison of harvest and processing techniques for fat grafting and adipose stem cell isolation. Plast Reconstructive Surgery 2013;132(2):351-361.

2. Coleman SR. Structural fat grafts: The ideal filler? Clinic Plastic Surgery 2001;28(1):111-119.

3. Arias-Gallo J, Chamorro-Pons M, Avendano C. Influence of acidic fibroblasts growth factor on bone regeneration in experimental cranial defects using spongostan and bio-oss as protein carriers. Journal of Craniofacial Surgery 2013;24(5):1507-1514.

4. Spotnitz WD, Burks S.Hemostats, sealants, and adhesives: components of the surgical toolbox. Transfusion 2008;48(7):15021516.

5. Kuran I, Tumerdem B. A new simple method used to prepare fat for injection. Aesthetic Plastic Surgery 2005;29(1):18-22.

6. Gir P, Brown SA, Oni G. Fat grafting: evidence based review on autologous fat harvesting, processing, reinjection, and storage. Plastic Reconstructive Surgery 2012;130(1):249-258. 